$\$=$

\title{
Colorectal carcinoma in Sudanese patients
}

\author{
Mutaz M Mohammed ${ }^{1 *}$, A magid M Musaad ${ }^{2}$, ElsaggadEltayeb ${ }^{3}$, Muataz A. elaziz ${ }^{4}$ \\ ${ }^{I}$ MD general surgery, Ibn Sina hospital \\ ${ }^{2}$ FRCS, Professor of GIT surgery, Omdurman Islamic University \\ ${ }^{3} \mathrm{MD}$, Associate professor of GIT surgery, Al-Neelain University \\ ${ }^{4}$, FRCS, Associate professor of GIT surgery, Al-Neelain University \\ *Corresponding author E-mail:mutazidres@yahoo.com
}

\begin{abstract}
Background: Cancer is an increasingly important public health problem in developing countries, including Sudan. The burden of colorectal cancer in Sudan is unknown, mainly because of lack of statistics or under reporting. Colorectal carcinoma is the most common malignancy of the GI tract, unfortunately there are few statistical data regarding colorectal cancer, its geographical, ethnic distribution, \& pattern of behavior in Sudan.

Objective: To assess pattern of presentation, management, \& postoperative complication of colorectal carcinoma patients who presented to Ibn Sina hospital.

Patients and method: This is a descriptive study between (2012-2014); a prospective \& retrospective study of histological confirmed cases of colorectal cancer was conducted at Ibn Sina hospital.

Result: Sixty three patients were included within the study. The mean age of the patients was $50.5( \pm 11.7)$. The median duration until presentation was 10 months. Left-side colonic carcinoma was $81 \%$ represented the majority of cases with Rectosigmoid cancer account for $74 \%$ of all cases. The most common presenting symptoms were alteration of bowel habits $90.5 \%$ and rectal bleeding $84 \%$. Anemia was reported in $62.5 \%$ of right side colonic carcinoma while only in $23.5 \%$ of left colonic carcinoma. CEA was found positive in $85.3 \%$ $(\mathrm{n}=35)$. Postoperative morbidity in this study was $38 \%$. All tumors were adenocarcinoma, and $65 \%$ of the sample were advanced "Duke's stage C \& D."

Conclusion: A younger age group with late presentation and advance disease, making the possibility of cure difficult if we take into consideration the scarcity of the resources.
\end{abstract}

Keywords: Colorectal Cancer; Clinicopathological Pattern of Presentation; Adenocarcinoma; Postoperative Morbidity; Mortality.

\section{Introduction}

In the World Health Organization bulletin, report No.804 of 1990, it was reported that over 50 percent of cancer victims live in poor nations which have less than 10 percent of the resources for cancer care and control.(AO et al. 2013)

Colorectal carcinoma is the most common malignancy of the GI tract, unfortunately there is few statistical data regarding colonic tumor \& its geographical, racial distribution in Sudan. A few studies conducted in Sudan showed a different disease pattern it tends to affect younger population than internationally reported.(AbdallaA \& Randa 2007; Abdelrahim MI 2010). That mean the targeted population for screening should be younger than the western counterpart. This type of information is vital for implementation of screening program, setting protocols, \& establishing national guidelines, hence improving patient's outcome and overall the heath service. The spectrum of presentation of colonic tumor is wide ranging from relatively healthy patient with few symptoms to life-threatening bowel obstruction with risk of perforation \& fecal peritonitis. Colorectal cancer occurs in hereditary, sporadic, or familial forms; hereditary forms of colorectal cancer have been extensively described and are characterized by family history, young age of the onset, and the presence of other specific tumors and defects.(Charles et al. 2009). In this study, colorectal cancer patients presented to Ibn Sina hospital were studied, structured data sheet used to analyze the demographic data, pattern of presentation, and therapeutic intervention that offered to the patients.

\section{Methods}

\subsection{Study design and setting}

Between (2012-2014), a prospective \& retrospective study of histological confirmed cases of colorectal cancer was conducted at Ibn Sina hospital. Ibn Sina hospital is located in Mohammed Najeeb St, Khartoum, one of few tertiary hospitals that provide advance services in gastrointestinal surgery, equipped with endoscopy unit for both elective \& emergency (Mohamed Salih center) cases, medical laboratory, \& pathology lab, in addition to otolaryngology, urology, renal, vascular unit, \& renal transplant center. The details of patients were retrieved from patients' files kept in the medical record department, the surgical wards, operating theatre, and histopathology laboratory. Information retrieved included socio-demographic data, clinical presentation, anatomical site, gross appearance, tumor stage, histopathological type and grade, presence of metastasis (nodal, distant and peritoneal), treatment modalities and outcome and follow-up. The anatomical subsite were categorized as cecum, ascending colon, transverse colon, 
descending colon, sigmoid, rectum, \& multiple synchronous carcinoma, then right $\&$ left sided cancer used in analysis in relation to proximal or distal location relative to the splenic flexure, the assumption is right \& left-side carcinoma follow broadly different molecular pathways of carcinogenesis.(Iacopetta 2002)

The diagnosis of colorectal cancer was performed by colonoscopy \& histopathology examination of colonoscopy specimens. Preoperative staging was conducted by either CT scan and/or MRI Base line CEA was performed for the majority of patients to be used in follow up. Full assessment of every patient by anesthesia team before surgery \& relevant investigation ordered to define patient's co morbidities. Final disease stage derived from the intraoperative assessment \& histopathological assessment of the surgical specimens.

\subsection{Ethical consideration}

Ethical approval to conduct the study was sought from Research ethical committee of Sudan Medical Specialization Board, \& hospital administration before the commencement of the study.

\section{Result}

A total of 63 patients included. The mean age of the patients in this study was $50.5( \pm 11.7)$, (Fig. 1) range between $(18-75$ years), the total number of patients below 48 years was $40 \%(n=25)$. Male to female ratio was appriximately1:1.

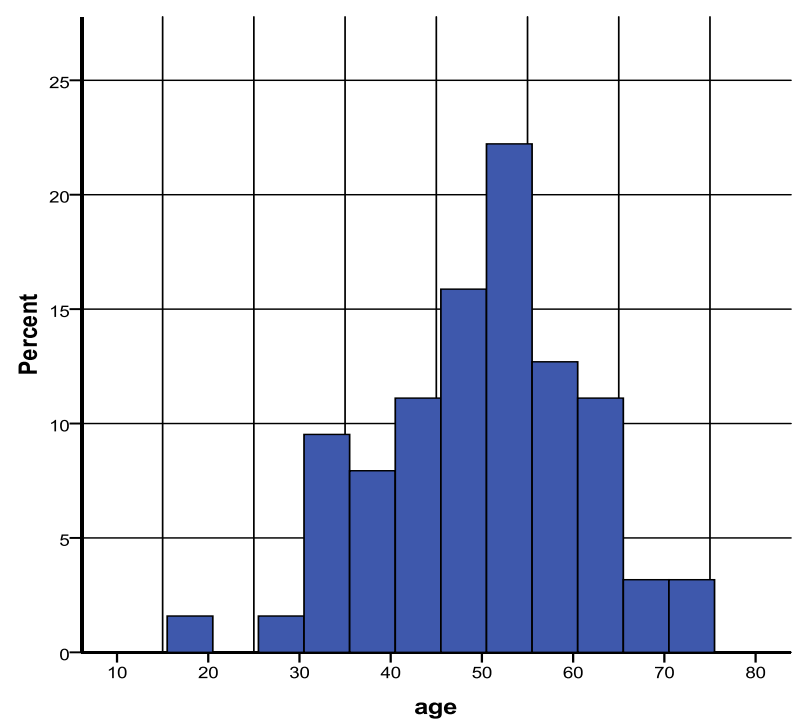

Fig. 1: Age Distribution among the Sample.

Geographic distribution showed that most of the patients presented from Khartoum state $33.3 \%(n=21)$, followed by northern state \&Kurdufan state $14.3 \%(\mathrm{n}=9)$ each, then Darfur state $11 \%(\mathrm{n}=7)$. The median duration of symptoms was 10 months. The most common presenting symptoms in this study was alteration of bowel habits $90.5 \%(n=57)$, rectal bleeding occurred in $84 \%(n=53)$, passage of mucus in $78 \%(n=49)$, and tenesmus in $63.5 \%(n=43)$, while abdominal pain presented in $54 \%(\mathrm{n}=34)$, anorectal pain in $43 \%(n=27)$, abdominal mass in $19 \%(n=12)$, abdominal distension in $20.6 \%(\mathrm{n}=13)$, and and fecal incontinence in $8 \%(\mathrm{n}=5)$, while less than $6 \%(n=4)$ presented with malena. The General manifestation of malignancy (anorexia, weight loss) were found in $63.5 \%(n=40)$. Anemia reported in $32 \%$ of patients $(n=20)$. (table $1)$.

The commonest site of the malignancy was found in the left side of the colon in $81 \%(n=51)$, right side carcinoma in $12.7 \%(n=8)$, while multiple synchronous carcinoma in $6.3 \%(\mathrm{n}=4)$ of patients. (Fig.2) Anemia was reported in $62.5 \%(\mathrm{n}=5)$ of right side colonic carcinoma while only in $23.5 \%(\mathrm{n}=12)$ of left colonic carcinoma.
Table 1: Symptoms Profile of the Sample

\begin{tabular}{lll}
\hline symptoms & Count & Column N \% \\
\hline Rectal bleeding & 53 & $84.1 \%$ \\
Alteration of bowel habit & 57 & $90.5 \%$ \\
Abdominal pain & 34 & $54.0 \%$ \\
Difficulty in defecation & 39 & $61.9 \%$ \\
Abdominal mass & 12 & $19.0 \%$ \\
Abdominal distension & 13 & $20.6 \%$ \\
Anorectal pain & 27 & $42.9 \%$ \\
Fecal incontinence & 5 & $7.9 \%$ \\
Malena & 4 & $6.3 \%$ \\
Passage of mucus & 49 & $77.8 \%$ \\
Tensmus & 40 & $63.5 \%$ \\
General manifestation of malignancy & 40 & $63.5 \%$ \\
Present with anemia & 20 & $31.7 \%$ \\
Total number of patients & & 63 \\
\hline
\end{tabular}

Those patients who might poses genetic predisposition with family history of colonic malignancy among their $1^{\text {st }}$ degree relative were $8 \%(n=5)$, and only one patient $(1.6 \%)$ was presented with colorectal cancer after being diagnosed with familial adenomatous polyposis. Only one patient had history of inflammatory bowel disease. $(1.6 \%)$
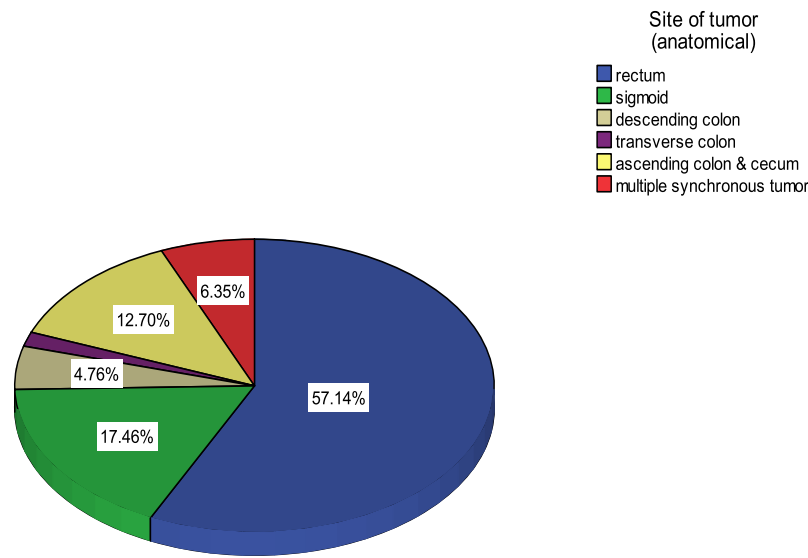

Fig.2: Subsite Distribution of the Colorectal Cancer.

Unfortunately CEA was performed in $65 \%(n=41)$ of patients, among those was found positive in $85.3 \%(n=35)$, negative in $14.7 \%$ $(\mathrm{n}=6)$.

All patients had a preoperative staging either by CT scan and/or MRI, and they showed that there was lymphadenopathy in $46.8 \%$ $(\mathrm{n}=29)$, and liver metastasis in $8 \%(\mathrm{n}=5)$.

Patients who received neoadjuvent therapy in this sample were $12.7 \%(\mathrm{n}=8)$.

APR was the most conducted surgery in $37 \%(n=23)$, colostomy in $9.5 \%(\mathrm{n}=6)$, right hemicolectomy\& sigmoid colectomy in $8 \%$ $(n=5)$ each, left hemicolectomy, high anterior resection, \&low anterior resection with ileostomy in $6.3 \%(\mathrm{n}=4)$ each, and low anterior resection without ileostomy in $4.8 \%(n=3)$.

The most frequent intraoperative finding was lymphadenopathy $66 \%$ $(n=41)$, followed by invasion to the surrounding organ in $35.5 \%$ $(n=22)$, peritoneal seeding in $14.5 \%(n=9)$, liver metastasis in $9.6 \%$ $(n=6)$, and ascites in $8 \%(n=5)$. The most frequent invaded structures among the tumor exhibit invasion $(n=22)$, was lateral abdominal wall invasion reported in $31.8 \%(\mathrm{n}=7)$, uterus \& cervix in $22.7 \%(n=5)$, small bowel in $13.6 \%(n=3)$.

Postoperative morbidity in this study was $38 \%(n=24)$, the reported complications were bleeding in $12.7 \%(\mathrm{n}=8)$, wound infection in $23.8 \%(\mathrm{n}=15)$, wound dehiscence in $1.6 \%(\mathrm{n}=1)$, and anastomotic leak $6.3 \%(n=2)$ in which anastomosis was constructed $(n=32)$. Postoperative ileus \& mechanical intestinal obstruction occurred only in $6.3 \%(n=4)$ each. (Fig.3). 


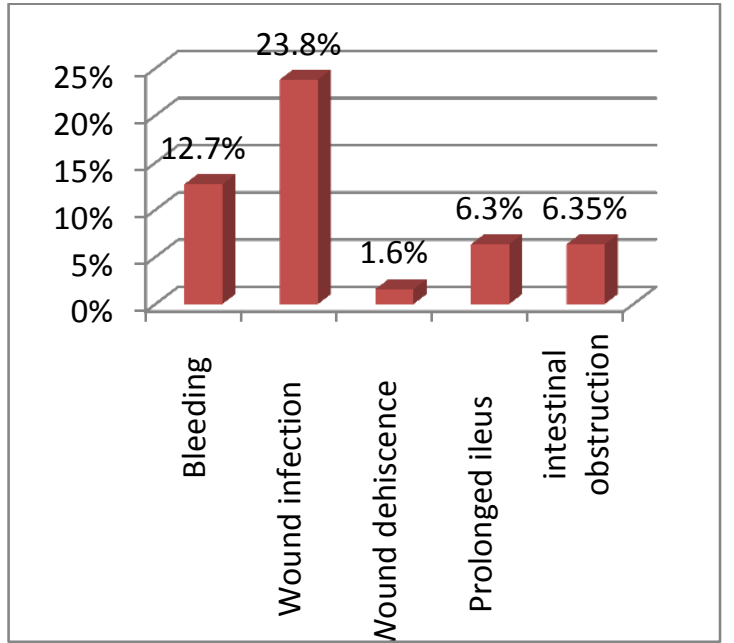

Fig.3: Postoperative Morbidity.

The result of the specimens were adenocarcinoma in most of the patients, it was moderately differentiated adenocarcinoma in $52.5 \%$ $(\mathrm{n}=31)$, poor differentiated in $32.2 \%(\mathrm{n}=19), \&$ well differentiated in $15.3 \%(\mathrm{n}=9)$.

Depending on Duke's staging system, the result was duke's A in $3.3 \%(n=2)$, stage B in31.7\% ( $n=19)$, stage $C$ in $46.7 \%(n=28)$, and stage $\mathrm{D}$ in $18.3 \%(\mathrm{n}=11)$. (Fig.4).

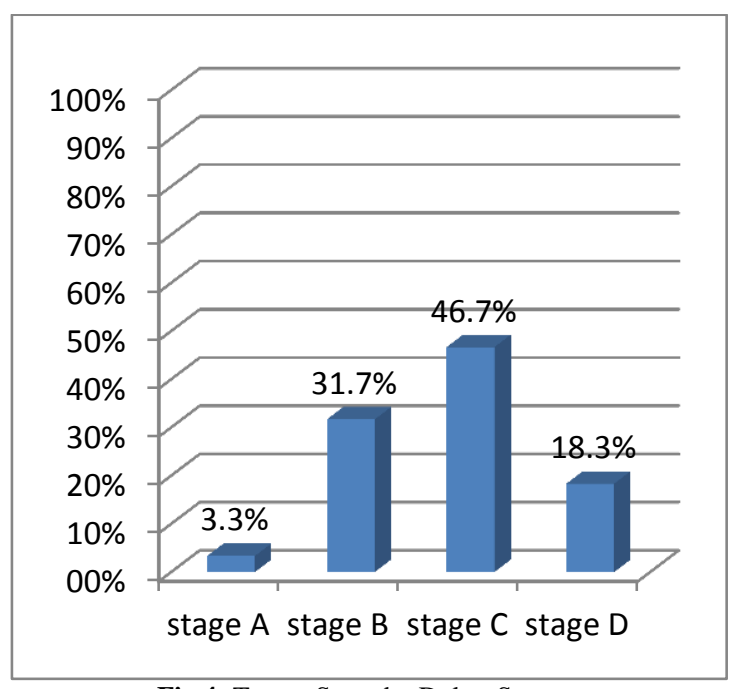

Fig.4: Tumor Stage by Dukes System.

One month mortality in this study was $6.3 \%(n=4)$.

\section{Discussion}

Colorectal cancer in Sudan was always a dilemma, Hicky 1958 in his article with the heading of Malignant Epithelial Tumors in the Sudanese stated, "These growths are of considerable frequency in Sudanese as compared with most other African statistics" \& this can be the result of some endemic parasitic infestation, but this was not proved.(Hickey 1959). In Malik et al, cancer of alimentary tract about half of the cases were anorectal cancer, \& $15 \%$ colonic cancer.(Malik et al. 1976)

In 2009 the first National Population-based Cancer Registry (NCR) was established in Sudan, they report in their First Data (20092010 ) that colorectal cancer (rate $=7.1$ per 100, 000) which make it number five among the most common cancers. (Saeed et al. 2014) The mean age of patients in this study was relatively young 50.5 yr. $40 \%$ of the studied patients were below age of 48 year, a percentage higher than (2 to 6\%) reported in western literature.(Charles et al. 2009) Only 20\% of our sample were 60 years or above. $5^{\text {th }}$ decade is the most frequent age group affected in international literature.(Charles et al. 2009). The modal age group in our study reported between 50-60 years. This observation was not made only from this study but since early reported studies done in Sudan among colorectal cancer patients showed it tend to affect younger age group with aggressive pattern of disease.(AbdallaA \& Randa 2007; Abdelrahim MI 2010; Elmasri \& Boulos 1975; Hickey 1959; Malik et al. 1976) Interestingly similar studies conducted in neighbor African countries showed similar figures, Phillipo L Chalya et al study of colorectal cancer in Tanzania,(Chalya et al. 2013) the most affected age group was $40-50, \& 38 \%$ of his studied population were below the age of 40 years. This might be explained by the demographic variation of population between western countries \& African one.

Regarding geographic distribution most of the patients presented from Khartoum state $33.3 \%(n=21)$, this due to high population density of Khartoum \& location of Ibn Sina hospital.

Most of the patients presented late, $64 \%$ of the studied sample presented after 6 months \& this is reflected on disease stage as it is shown in histopathology report below, \& ultimately cancer prognosis \& survival. The median duration until presentation was 10 months, duration Calls for concern if we compare it to a median duration of only $14 \mathrm{wk}$ in D Smith study (Symptomatic presentation of early colorectal cancer in U.K).(Smith et al. 2006)

Symptoms are common and prominent in late colon cancer when the prognosis is poor but are less common and less obvious early in the disease.(Cappell 2008) The most common presenting symptoms in this study was alteration of bowel habits $90.5 \%(\mathrm{n}=57)$, rectal bleeding occurred $84 \%(\mathrm{n}=53)$, passage of mucus $78 \%$ $(n=49)$, tensmus $63.5 \%(n=43)$, and abdominal pain54\% $(n=34)$, these symptoms complex reflect the predominance of left side colonic tumor which account for $81 \%(n=51)$, it would appear that alteration of bowel habit \& rectal bleeding in adult warrant urgent attention rather than discharging the patients without full workup, this trend correlate with Ahmed A Abdalla et al study of rectal carcinoma in Madani which showed similar figures, (Abdalla et al. 2015) but were different from literature which shows a lower frequency rates,(Cappell 2008) this may be due to asymptomatic \& early cancer cases represent substantial percentage in developed countries.(Society 2011)

In Ahmed A Abdalla \& Mohamed Toum Musa colorectal cancer in Khartoum hospital $35 \%$ presented with intestinal obstruction,(AbdallaA \& Randa 2007) which correlate with advanced disease, also Popoola et al study in Lagos found a similar trend.(Popoola et al. 2012) In our study most of the patients present electively, although finding of near obstructing tumor during surgeries was not uncommon.

Comparing symptomatology of left and right side carcinoma, rectal bleeding $92 \%$ ( $\mathrm{p}$ value $=0.001)$, tensmus $70.6 \%(\mathrm{p}$ value $=$ 0.006 ), and anorectal pain $51 \%$ ( $\mathrm{p}$ value $=0.019$ ) were more discriminative of left sided carcinoma and reached significance. While abdominal mass $87.5 \%$ ( $p$ value $=0.001$ ) and anemia $62.5 \%$ ( $\mathrm{p}$ value $=0.014)$ where more common and reached significance in right side carcinoma, and this correlate well with the literature.(Cappell 2008; Richman \& Adlard 2002)

It's well known that the incidence of colonic cancer increase with the positive family history, relative risk of $(2.25)$ in Louise E Johns \& Richard S Houlston meta-analysis of familial colorectal cancer risk.(Johns \& Houlston 2001). As reported from the literature familial colorectal cancer account for almost $10-15 \%$ of all colorectal cancer.(Charles et al. 2009) In our study the figure is lower $8 \%(n=5), \&$ one patient $1.6 \%$ presented with colorectal cancer after being diagnosed with $\mathrm{FAB}$, this is higher than some African studies in Phillipo L Chalya et alreported positive family history in 5.4\% of cases.(Chalya et al. 2013).

Regarding the site of the carcinoma, left-sidecolonic carcinoma was $81 \%(n=51)$, right side carcinoma in $12.7 \%(n=8)$, while multiple synchronous carcinoma in $6.3 \%(n=4)$ of patients. Rectosigmoid cancer account for $74 \%$ of all cases which is higher than reported throughout the literature (Cucino et al. 2002). worldwide rectosigmoid carcinoma is the most frequent subsite affected, but in our study, we reported a high percentage (74\%) in comparison 
to literature, this finding is comparable with similar studies done in Sudan,(AbdallaA \& Randa 2007; Abdelrahim MI 2010; Elmasri $\&$ Boulos 1975) even malik et al 1958 point to frequency of anorectal carcinoma, this reflected no time trend change in sub-site distribution of colorectal cancer, in contrary to recent western studies which point to proximal migration phenomena, \& some author find right side carcinoma in up to $40 \%$.(Miller et al. 2000; Papagiorgis et al. 2014).

Regarding synchronous carcinoma, the reported literature incidence rate $(3-5 \%)$ is not far from ours 6.3\%.(Souba et al. 2007) This figure may be overestimation of the true figure, because Ibn Sina is a tertiary center \& difficult cases usually referred to it. According to colonoscopy report the most common tumor pattern is fungating mass in $55.7 \%(\mathrm{n}=34)$. Among left sided carcinoma fungating was the most reported in $49 \%(\mathrm{n}=24)$ and annular carcinoma in $42 \%(n=21)$, while right side carcinoma fungating mass was the most frequent reported in $87.5 \%$. This approximately follow the usual pattern of polypoid tumor in the right colon and constricting lesions in the left side, where intestinal obstruction is common presentation if left untreated.(Bailey et al. 2013)

In 1965, Gold and Freedman described an oncofetal antigen expressed in human fetal colonic tissues and in colonic carcinomas but not in adult colon; they named it carcinoembryonic antigen (CEA).(Duffy 2001) Unfortunately not all patients data contain CEA level; it was performed in $65 \%(\mathrm{n}=41)$ of patients, among those was found positive in $85.3 \%(n=35)$, negative in $14.7 \%$ $(n=6)$. The sensitivity of CEA for early colon cancer patients is low and increases with an increasing stage of the disease.(Duffy 2001) An elevated serum level preoperatively is a poor prognostic indicator; the higher the serum level the more likely the cancer is extensive and will recur postoperatively.(Cappell 2008) A finding which we replicated it in this study, only $50 \%$ of stage A disease had elevated CEA level in comparison to $95 \%, 100 \%$ of stage C \& $\mathrm{D}$ respectively (Pearson Chi-Square $=6.9$ with $\mathrm{p}=0.04$ ) reaching significance.

Standard preoperative evaluation done to all patients to assess their preoperative stage either by CT scan or MRI, or both. Lymphadenopathy detected by CT scan in $46.8 \%(\mathrm{n}=29)$, liver metastasis in $8 \%(\mathrm{n}=5)$, Comparing these results with the postoperative specimens study CT scan has $70 \%$ sensitivity in detecting lymph node involvement; this finding doesn't correlate with the $45 \%$ sensitivity for lymph node involvement reported by many authors.(Freeny et al. 1986; Thoeni 1997) This might be explained by sample bias, in our sample the majority of patients were advanced at presentation making detection of abnormality easier. If the mesorectum around a rectal cancer is involved or threatened (only 1 to $2 \mathrm{~mm}$ of clearance), there is a very high likelihood of local recurrence and a poor prognosis.(Charles et al. 2009) This circumferential or radial margin (CRM) is probably best assessed preoperatively by MRI. In our sample (rectal cancer $n=36$ ) CRM involved in $37 \%(n=13)$, not involved in $28.6 \%(n=10)$, not assessed in $34.3 \%(n=13)$. Tools used to assess the Circumferential margin were MRI in $70 \%(n=16)$ CT scan in $30 \%(n=7)$. In Ahmed A Abdalla et al study of rectal carcinoma in Madani MRI was performed for $52.8 \%$ while $47.2 \%$ were assessed by CT scan.(Abdalla et al. 2015) Nowadays endorectal US \& MRI used to accurately stage patients with rectal carcinoma, to determine who might benefit from preoperative neoadjuvent therapy, \& who will undergo sphincter saving procedure.(Charles et al. 2009). In this respect we are lagging behind, possible explanation is relative paucity of MRI machine, mostly in private hospitals, \& high cost. An endorectal $\mathbf{U} / \mathbf{S}$ is operator dependant and need training.

Patients who received neoadjuvent chemotherapy in this sample were $12.7 \%(\mathrm{n}=8)$, their initial staging showed locally advance disease, $42 \%$ downstaged to stage A \& B, \& 57\% didn't respond but this number is too small to be correctly statistically analyzed. Ahmed A Abdalla et al study of rectal carcinoma in Madani addressed this issue \& showed a promising result.(Abdalla et al. 2015)

APR was the most conducted surgery in $37 \%(n=23)$, reflecting the high proportion of rectal cancer in the sample. Worldwide introduction of neoadjuvent therapy and stapler anastomosis resulted in performing more sphincter preserving operations; unfortunately, the limited capabilities, and absence of national guidelines for the neoadjuvent group, explain the high rate of Abdominoperineal resection.

Regarding intraoperative finding, the most frequent finding was lymphadenopathy $66 \% \quad(\mathrm{n}=41)$, followed by invasion $35.5 \%$ $(n=22)$, the most frequent invaded structure $(n=22)$ is lateral $a b-$ dominal wall $11.1 \%(n=7)$, uterus \& cervix in $8 \%(n=5)$, small bowel in $4.7 \%(n=3)$. Liver metastasis in $9.5 \%(n=6)$, no specific cancer site showed predilection for liver metastasis.

Despite refinements in surgical techniques, bowel preparation patterns, prophylactic antibiotics, and postoperative care in recent decade, colorectal surgery still associated with serious complications. Postoperative morbidity in this study was $38 \%(n=24)$, in agreement with Arnaud Alves in French study.(A et al. 2005) The most frequent complication was SSI $23.8 \%(\mathrm{n}=15)$; most was superficial SSI that was managed with dressing \& antibiotics, other authors reported a less frequency.(Khan et al. 2011) Bleeding was the second most occurring complication $12.7 \%(\mathrm{n}=8)$, only one patient needed return to OR. Postoperative ileus \& mechanical intestinal obstruction in $6.3 \%(\mathrm{n}=4)$ each.

Perhaps the most devastating complication that can occur following colonic surgery is anastomotic dehiscence. To correctly analyze the anastomotic dehiscence rate, we excluded those patients in whom no anastomosis was constructed (APR, colostomy); the remaining patients were $(n=32)$, anastomotic dehiscence reported in $6.3 \%(\mathrm{n}=2)$, both occurred in rectosigmoid region. Anastomotic dehiscence rate varied widely between studies \& several factor controlling its frequency (age, site of the tumor, nutritional status, and emergency presentation versus elective one, overall physiological status). Some authors described high anastomotic dehiscence rate $11 \%$ in rectal surgery, while others described a lower rates 3.2\%.(Khan et al. 2011; Paun et al. 2010) in spite of this variations all studies concordant in predominance of anastomotic dehiscence among left sided carcinoma, and the current study was able to validate this observation.

An increasing proportion of sphincter-saving operations is used in modern rectal cancer surgery; this had led to recognition of relatively new complication termed anterior resection syndrome; a well-known and well-described complication after restorative rectal surgery with a straight anastomosis. It occurs due to loss of rectal reservoir, colonic dysmotility, \& sphincter damage. In our study, 11 patients underwent anterior resection; frequent motions were the most frequent symptoms $70 \%(\mathrm{n}=7)$, fecal incontinence in $20 \%(\mathrm{n}=2)$, urgency in $10 \%(\mathrm{n}=1)$. It is widely accepted that up to $90 \%$ of such patients will subsequently have a change in bowel habit, ranging from increased bowel frequency to fecal incontinence or evacuatory dysfunction, this study shows also a similar trend.(Bryant et al. 2012; Desnoo \& Faithfull 2006)

All the sample patients were adenocarcinoma, regarding grade, moderate differentiated occurred in $52.5 \%(n=31)$, poor differentiated into $32.2 \%(\mathrm{n}=19), \&$ well differentiated into $15.3 \%(\mathrm{n}=9)$

The final histopathology reports showed that $65 \%$ of the sample were advance Tumor. Duke's stage A reported in only 3.3\% $(\mathrm{n}=2)$, stage $B$ in $31.7 \%(n=19)$, stage $C$ in $46.7 \%(n=28)$, stage $D$ in $18.3 \%$ $(\mathrm{n}=11)$. A similar trend also reported in some African \& regional studies, (Chalya et al. 2013) but in countries where cancer screening program is active early stage disease comprise $40 \%$ of the new cases.(Society 2011).

Factor associated with advance disease in this study; tumor grade correlates with advance disease (Pearson Chi-Square $=18.9$ with $\mathrm{p}$ $=0.004$ ) reaching significance, in agreement with the literature.(Willett 2001) abdominal pain (Pearson Chi-Square $=5.5$ with $\mathrm{p}=0.027$ ) reaching significance, possible explanation of this association is when the tumor attain a large size it usually gives rise to obstructive symptoms, of which abdominal pain is a major one, in agreement with D Smith study.(Smith et al. 2006). Right side tumors are usually occult $\&$ manifest late with anemia symptoms, while left-side tumors manifest early with symptoms of rectal bleeding $\&$ altered bowel habits, the former tends to present 
with advance disease, (Gonzalez et al. 2001) in our study, although $37.5 \%$ of right side \& only $16 \%$ of left-side tumor were stage $\mathrm{D}$, this is failed to reach significance.

Postoperative mortality defined as death within 30 days after the operation, in this study was $6.3 \%(\mathrm{n}=4)$. The leading complication to death was mechanical intestinal obstruction \& sepsis. Other authors which reported 30-day mortality as the main endpoint of their studies showed that mortality in this period of time ranged between 1-11.9\%.(Damhuis et al. 1996; Davila et al. 2005; Nickelsen et al. 2005)

\section{Conclusion}

The current trend of colorectal cancer in this study was a young age group with advance disease. Rectal bleeding \& altered bowel habits were the commonest presenting symptoms, \& the majority of patients came from distant areas. The challenge, therefore, must be to reduce the number of cancers presenting late and swing the pendulum in favor of early cancer detection. Implementation of screening program, Establishment of national based protocol for colorectal cancer management, \& guideline for referral system between health centers \& tertiary hospital can improve the outcome.

\section{Acknowledgements}

There was no grant or other financial support for this article.

\section{References}

[1] A, A. et al., 2005. Postoperative mortality and morbidity in french patients undergoing colorectal surgery: Results of a prospective multicenter study. Archives of Surgery, 140(3), pp.278-283. Available at: http://dx.doi.org/10.1001/archsurg.140.3.278.

[2] Abdalla, A.A.E., Alawad, A.A.M. \& Ali, H.A.M., 2015. Pathologic response after neoadjuvant chemoradiotherapy in Sudanese patients with locally advanced rectal adenocarcinoma. International journal of medicine, 3(2), p.53. Available at: http://dx.doi.org/10.14419/ijm.v3i2.4836.

[3] AbdallaA, M.M. \& Randa, Z., 2007. Presentation of Colorectal Cancer in Khartoum Teaching Hospital. Sudan Journal of Medical Sciences, 2, pp.263-267.

[4] Abdelrahim MI, S.S., 2010. The pattern and prevention of Colorectal cancer in Sudan. smj.

[5] AO, P. et al., 2013. Prevalence and Pattern of Cancers among Adults Attending a Tertiary Health Institution in Lagos, Nigeria. Journal of Dental and Medical Sciences, 6(3), pp.6873.http://dx.doi.org/10.9790/0853-0636873.

[6] Ayyub, M.I. et al., 2002. Clinicopathological trends in colorectal cancer in a tertiary care hospital. Saudi medical journal, 23(2), pp.160-163.

[7] Bailey, H. et al., 2013. Bailey \& Love's short practice of surgery 26th ed., CRC Press.

[8] Bryant, C.L. et al., 2012. Anterior resection syndrome. The lancet oncology, 13(9), pp.e403-e408.http://dx.doi.org/10.1016/S14702045(12)70236-X.

[9] Cappell, M.S., 2008. Pathophysiology, clinical presentation, and management of colon cancer. Gastroenterology Clinics of North America, 37(1), pp.1-24. Available at: http://dx.doi.org/10.1016/j.gtc.2007.12.002.

[10]Chalya, P.L. et al., 2013. Clinicopathological patterns and challenges of management of colorectal cancer in a resource-limited setting: a Tanzanian experience. World J Surg Oncol, 11(1), pp.88-97. Available at: .http://dx.doi.org/10.1186/1477-7819-11-88

[11]Charles, B.F. et al., 2009. Schwartz's principles of surgery, McGrawHill, Health Pub. Division.

[12]Cucino, C., Buchner, A.M. \& Sonnenberg, A., 2002. Continued rightward shift of colorectal cancer. Diseases of the colon \& rectum, 45(8), pp.1035-1040. Available at: http://dx.doi.org/10.1007/s10350004-6356-0.

[13] Damhuis, R., Wereldsma, J. \& Wiggers, T., 1996. The influence of age on resection rates and postoperative mortality in 6457 patients with colorectal cancer. International journal of colorectal disease 11(1), pp.45-48. Available at: http://dx.doi.org/10.1007/BF00418856.
[14]Davila, J.A. et al., 2005. Postoperative 30-day mortality following surgical resection for colorectal cancer in veterans: changes in the right direction. Digestive diseases and sciences, 50(9), pp.1722-1728. Available at: http://dx.doi.org/10.1007/s10620-005-2925-x.

[15]Desnoo, L. \& Faithfull, S., 2006. A qualitative study of anterior resection syndrome: the experiences of cancer survivors who have undergone resection surgery. European journal of cancer care, 15(3), pp.244-251. Available at: http://dx.doi.org/10.1111/j.13652354.2005.00647.x.

[16]Duffy, M.J., 2001. Carcinoembryonic antigen as a marker for colorectal cancer: is it clinically useful? Clinical chemistry, 47(4), pp.624-630.

[17]Elmasri, S. \& Boulos, P., 1975. Carcinoma of the large bowel in the Sudan. British Journal of Surgery, 62(4), pp.284-286. Available at: http://dx.doi.org/10.1002/bjs.1800620408.

[18]Freeny, P.C. et al., 1986. Colorectal carcinoma evaluation with CT: preoperative staging and detection of postoperative recurrence. Radiology, 158(2), pp.347-353. Available at: http://dx.doi.org/10.1148/radiology.158.2.3941861.

[19] Gonzalez, E.C. et al., 2001. Predictors of proximalvs. distal colorectal cancers. Diseases of the colon \& rectum, 44(2), pp.251-258. Available at: http://dx.doi.org/10.1007/BF02234301.

[20]Hickey, B.B., 1959. Malignant Epithelial Tumours in the Sudanese: Hunterian Lecture delivered at the Royal College of Surgeons of England on 13th March 1958. Annals of the Royal College of Surgeons of England, 24(5), p.303.

[21]Iacopetta, B., 2002. Are there two sides to colorectal cancer? International journal of cancer, 101(5), pp.403-408. Available at: http://dx.doi.org/10.1002/ijc.10635.

[22] Johns, L.E. \& Houlston, R.S., 2001. A systematic review and metaanalysis of familial colorectal cancer risk. The American journal of gastroenterology, 96(10), pp.2992-3003. Available at: http://dx.doi.org/10.1111/j.1572-0241.2001.04677.x.

[23] Khan, M., Bari, H. \& Raza, S., 2011. Early postoperative outcome after curative colorectal cancer surgery. Singapore medical journal, 52(3), pp.195-200.

[24]Malik, M., El Din, Z. \& El Masri, S., 1976. Cancer of the alimentary tract in the Sudan. A study of 546 cases. Cancer, 37(5), pp.25332542. Available at: http://dx.doi.org/10.1002/1097-0142(197605).

[25]Miller, A., Gorska, M. \& Bassett, M., 2000. Proximal shift of colorectal cancer in the Australian Capital Territory over 20 years. Australian and New Zealand journal of medicine, 30(2), pp.221-225. Available at: http://dx.doi.org/10.1111/j.1445-5994.2000.tb00811.x.

[26]Nickelsen, T., Jørgensen, T. \& Kronborg, O., 2005. Thirty-day mortality after surgery for colorectal cancer in Denmark. Colorectal disease, 7(5), pp.500-506. Available at: http://dx.doi.org/10.1111/j.1463-1318.2005.00793.x.

[27]Papagiorgis, P.C. et al., 2014. Proximal shift of colorectal cancer. A persistent phenomenon with multiple causes, patterns and clinical implications. Journal of BU ON.: official journal of the Balkan Union of Oncology, 19(3), p.605.

[28]Paun, B.C. et al., 2010. Postoperative complications following surgery for rectal cancer. Annals of surgery, 251(5), pp.807-818. Available at: http://dx.doi.org/10.1097/SLA.0b013e3181dae4ed.

[29] Popoola, A. et al., 2012. Clinico-Pathological Presentation of Colorectal Cancer Seen in Lagos State University Teaching Hospital. Macedonian Journal of Medical Sciences, 5(4), pp.411-415. Available at: http://dx.doi.org/10.3889/MJMS.1857-5773.2012.0245.

[30]Richman, S. \& Adlard, J., 2002. Left and right sided large bowel cancer. Bmj, $324(7343), \quad$ pp.931932.http://dx.doi.org/10.1136/bmj.324.7343.931.

[31] Saeed, I.E. et al., 2014. Cancer incidence in Khartoum, Sudan: first results from the Cancer Registry, 2009-2010. Cancer Medicine, 3(4), pp.1075-1084. Available at: http://dx.doi.org/10.1002/cam4.254.

[32]Smith, D. et al., 2006. Symptomatic presentation of early colorectal cancer. Annals of the Royal College of Surgeons of England, 88(2), p.185. Available at: http://dx.doi.org/10.1308/003588406X94904.

[33] Society, A.C., 2011. Colorectal Cancer Facts \& Figures 2011-2013.

[34]Souba, W.W. et al., 2007. ACS surgery: principles \& practice 6th ed. D. M. O. Bruce M. Brenner, ed., WebMD Professional Pub.

[35] Thoeni, R.F., 1997. Colorectal cancer. Radiologic staging. Radiologic Clinics of North America, 35(2), pp.457-485.

[36]Willett, C.G., 2001. Cancer of the lower gastrointestinal tract C. G. Willett, ed., PMPH-USA. 